\title{
Depressive Symptoms Are Associated With Higher Rates of Readmission or Mortality After Medical Hospitalization: A Systematic Review and Meta-analysis
}

\author{
Jenelle L. Pederson, MSc ${ }^{1}$, Lindsey M. Warkentin, $\mathrm{MSc}^{2}$, Sumit R. Majumdar, MD, MPH ${ }^{1,3}$, Finlay A. McAlister, MD, MSc ${ }^{1,4 *}$
}

${ }^{1}$ Division of General Internal Medicine, Faculty of Medicine and Dentistry, University of Alberta, Edmonton, Canada; 'Division of Surgery, Faculty of Medicine and Dentistry, University of Alberta, Edmonton, Canada; ${ }^{3}$ Alberta Diabetes Institute, Edmonton, Canada; ${ }^{4}$ The Patient Health Outcomes Research and Clinical Effectiveness Unit, Faculty of Medicine and Dentistry, University of Alberta, Edmonton, Canada.

Depressive symptoms during a medical hospitalization may be an overlooked prognostic factor for adverse events postdischarge. Our aim was to evaluate whether depressive symptoms predict 30-day readmission or death after medical hospitalization. We conducted a systematic review of studies that compared postdischarge outcomes by inhospital depressive status. We assessed study quality and pooled published and unpublished data using random effects models. Overall, one-third of 6104 patients discharged from medical wards were depressed (interquartile range, $27 \%-40 \%$ ). Compared to inpatients without depres- sion, those discharged with depressive symptoms were more likely to be readmitted $(20.4 \%$ vs $13.7 \%$, risk ratio $[R R]: 1.73,95 \%$ confidence interval $[\mathrm{Cl}]: 1.16-2.58)$ or die (2.8\% vs $1.5 \%$, RR: $2.13,95 \% \mathrm{Cl}: 1.31-3.44)$ within 30 days. Depressive symptoms were common in medical inpatients and are associated with an increased risk of adverse events postdischarge. Journal of Hospital Medicine 2016;11:373-380. (c) 2016 The Authors Journal of Hospital Medicine published by Wiley Periodicals, Inc. on behalf of Society of Hospital Medicine
Between $10 \%$ and $40 \%$ of patients are readmitted after being discharged from the hospital, ${ }^{1,2}$ and as many as another $25 \%$ return to the emergency department (ED) within 30 days. $^{3}$ This creates a substantial burden on the healthcare system. ${ }^{2}$ Various interventions have been tried to improve the quality of discharge transitions and reduce readmission rates, but results thus far have been inconsistent and generally disappointing. ${ }^{4-6}$ Targeted delivery of interventions to those at highest risk might improve the effectiveness of these efforts and reduce costs. However, current readmission risk assessment models are only moderately predictive, suggesting the presence of unrecognized risk factors. ${ }^{7,8}$

Active depression might represent a potentially modifiable independent predictor of adverse short-term hospital outcomes that is currently underutilized. Depression occurs in $5 \%$ to $58 \%$ of hospitalized adults, depending on how cases are defined. ${ }^{9,10}$ Depression is often underrecognized and undertreated in acute care clinical set-

*Address for correspondence and reprint requests: Finlay A. McAlister, MD, 5-134C Clinical Sciences Building, University of Alberta, 1135083 Avenue, Edmonton, Alberta, Canada T6G 2G3; Telephone: 780-4929824; Fax: 780-492-7277; E-mail: finlay.mcalister@ualberta.ca

Additional Supporting Information may be found in the online version of this article.

This is an open access article under the terms of the Creative Commons Attribution-NonCommercial-NoDerivs License, which permits use and distribution in any medium, provided the original work is properly cited, the use is non-commercial and no modifications or adaptations are made.

Received: July 29, 2015; Revised: December 1, 2015; Accepted: December 21, 2015

2016 Society of Hospital Medicine DOI 10.1002/jhm.2547

Published online in Wiley Online Library (Wileyonlinelibrary.com). tings, ${ }^{11}$ and relatively few readmission prediction models incorporate mental health related symptoms. ${ }^{12}$

Although several reviews have examined methods of screening for depression in hospitalized patients ${ }^{9}$ or the effectiveness of screening in primary care, ${ }^{13,14}$ to our knowledge no systematic review has examined the impact of depression on short-term prognosis after discharge from acute care. Therefore, the purpose of this systematic review was to summarize all studies that evaluated whether hospitalized medical patients with depressive symptoms are at higher risk of 30-day all-cause readmission or all-cause mortality after being discharged from the hospital.

\section{METHODS}

This study followed an a priori protocol developed according to PRISMA (Preferred Reporting Items for Systematic Reviews and Meta-Analyses) criteria. ${ }^{15}$

\section{Data Sources and Search Methods}

We searched the Cumulative Index to Nursing and Allied Health Literature, Ovid MEDLINE, Ovid Embase, and PsycINFO from inception to January 9, 2015, and the last 5 years of PubMed for full publications with any of the following Medical Subject Headings: "depressive disorder," “depression," "patient readmission," “interviews, psychological," "inpatients," with restrictions for peer-reviewed publication, humans, adults aged $\geq 18$ years, and the English language. Search strategies were developed with a librarian (available upon request). We manually searched reference lists of all included studies and relevant review articles and contacted content experts to identify additional publications. 


\section{Eligibility Criteria and Selection of Studies}

Two authors (J.L.P. and L.M.W.) independently screened full texts of all relevant articles for inclusion. Disagreements were resolved by consensus or a third reviewer (S.R.M.). We considered any original research that compared readmission or mortality after discharge for hospitalized medical patients (ie, general patients or subgroups thereof) with versus without depression identified by any validated depression measure, ${ }^{16}$ including any study design that incorporated at least 30-day follow-up postdischarge. We excluded studies that examined patients hospitalized in nonacute care settings or on surgical, psychiatric, obstetric, or intensive care services. We calculated Cohen's $\kappa$ coefficient to evaluate inter-rater agreement on study selection.

\section{Data Extraction}

Data were abstracted by 2 authors (J.L.P. and L.M.W.). Disagreements were resolved by consensus or a third reviewer (S.R.M.). We contacted authors of all included studies to obtain missing data. If unavailable, crude data were estimated from published survival curves employing validated techniques in $R$ (version 3.1.2; R Foundation for Statistical Computing, Vienna, Austria) and Digitizeit (http://www.digitieit.de; DigitizeIt, Braunschweig, Germany). ${ }^{17,18}$ We sought information on trial characteristics (country, type of hospital, inclusion and exclusion criteria, sample size, follow-up duration, attrition), participants (age, sex, ethnicity, level of education and social support, comorbidities, marital status), exposure ascertainment (self-report depression screening tool or diagnostic interview for depression), and outcomes (primary: 30-day all-cause readmission or mortality, secondary: 90-day all-cause readmission or mortality, $\mathrm{ED}$ visits, primary care physician $[\mathrm{PCP}]$ visits).

\section{Data Synthesis and Statistical Analysis}

Where possible, we calculated the pooled risk ratio (RR) with 95\% confidence interval $(95 \% \mathrm{CI})$ using a random effects models in Review Manager (RevMan) 5.3 (The Nordic Cochrane Centre, Copenhagen, Denmark). The random effects approach that we employed assumes heterogeneity (ie, underlying parameters vary between individual studies) and is distributed around a mean or "population average" effect, and results in more conservative (wider) confidence intervals, wherein larger cohorts (or studies with smaller standard errors) are given more weight. Heterogeneity was assessed using the $I^{2}$ statistic, with values of $<25 \%, 25 \%$ to $50 \%$, and $>50 \%$ representing low, moderate, and high heterogeneity. ${ }^{19}$ As per the guidance of Higgins et al., we did not a priori define any degree of heterogeneity that would preclude pooling of the data; the expectation would be that heterogeneity would be substantially higher pooling observational studies rather than randomized trials. ${ }^{19}$ Statistical significance was considered a 2 sided $P$ value $\leq 0.05$.

\section{Quality Assessment and Risk of Bias}

We assessed study quality using the 9-item NewcastleOttawa scale with 0 to 3,4 to 6 , and 7 to 9 stars considered low, moderate, and high quality, respectively, and criteria for external and internal validity, including group selection and comparability, outcome assessment, and adequacy of follow-up. ${ }^{20}$ Adjusted estimates published in individual reports (or obtained directly from authors) were compared wherever possible with unadjusted estimates to assess the degree of confounding. We generated funnel plots in RevMan 5.3 and conducted Egger tests using Stata 13 (StataCorp LP, College Station, TX) to assess for publication bias. $^{21}$

\section{RESULTS}

\section{Study Selection}

After removing duplicate publications, we identified 4066 reports and reviewed 133 reports in full text (see Supporting Figure 1 in the online version of this article). Despite our broad study inclusion criteria, we found only 35 longitudinal studies addressing this question. All 35 authors were contacted for additional outcomes data and other missing information (response rate of $34 \%$ ). We had to exclude 17 studies as they did not provide 30 or 90 -day post-discharge outcomes. Only 4 studies had published crude data for outcomes within 90 days, ${ }^{22-25}$ but after contact with authors, we received unpublished data for a further 7 studies $^{26-32}$ (including individual level data for 2 cohorts). ${ }^{31,32}$ We were able to estimate crude data from Kaplan-Meier curves for another 3 studies. $^{33-35}$ Another 4 studies did not collect the outcomes we were interested in individually. These studies were included in this systematic review but are not poolable in our models: 3 authors could only provide composite endpoint data, ${ }^{36-38}$ and 1 author provided unadjusted hazard ratios. ${ }^{39}$ Inter-reviewer agreement for inclusion was $80 \%$ (Cohen's $\kappa=0.60$ ).

\section{Characteristics of Included Studies}

The 18 studies ranged in size from 58 to 1418 patients; 13 were cohort studies and 5 included secondary data from randomized control trials. $22,27,30,34,36$ All studies ascertained depressive status by screening during index medical admission with either diagnostic interview or self-report questionnaires, although a variety of scales and definitions for depression were used (Beck Depression Inventory [BDI] in 6 studies, Geriatric Depression Scale in 5 studies, Patient Health Questionnaire in another 4 studies, Medical Outcomes Study-Depression Questionnaire in 1 study, and Center for Epidemiologic Studies Depression Scale in another study) (Table 1). Screening interviews were conducted mostly 


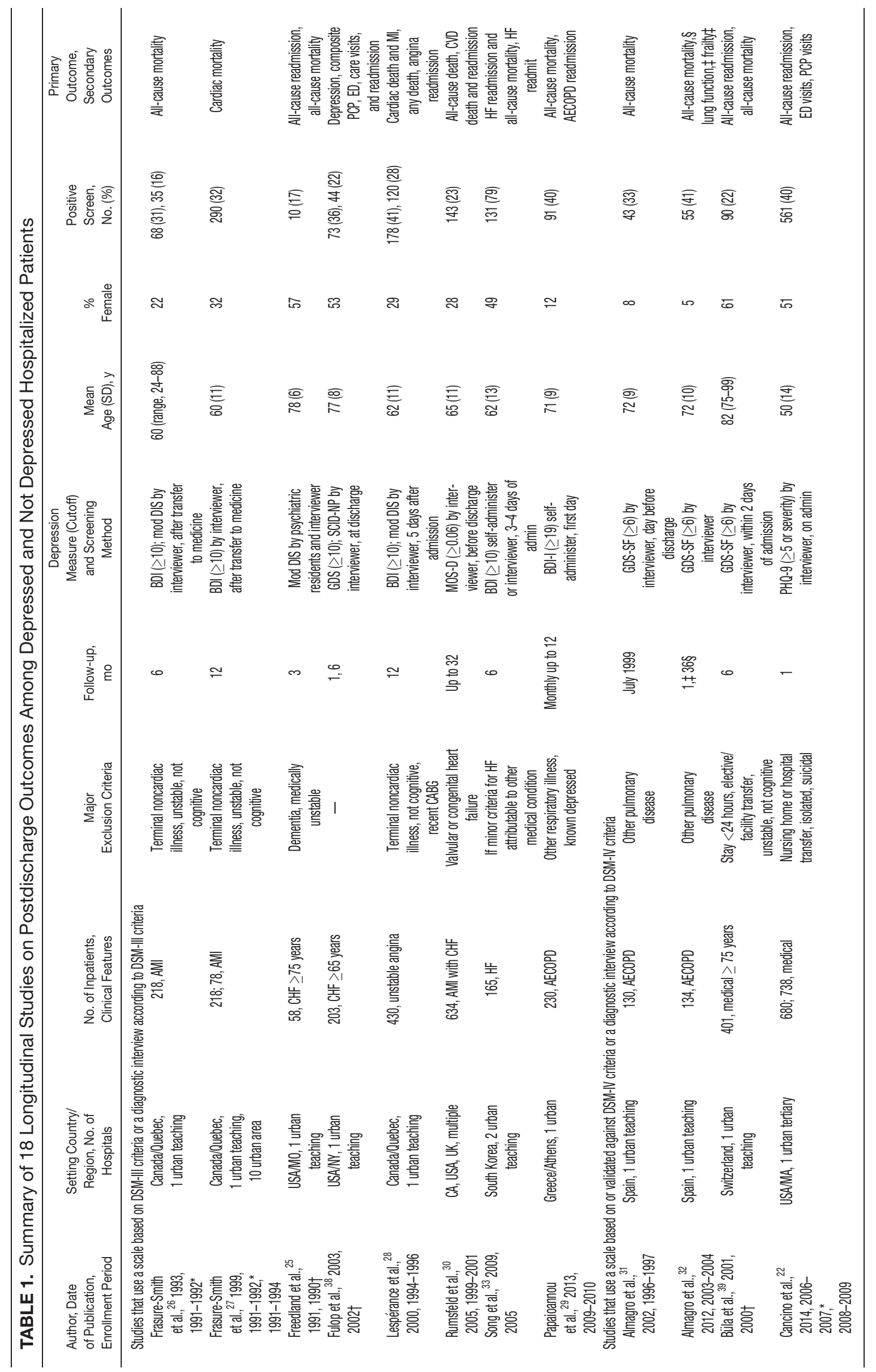




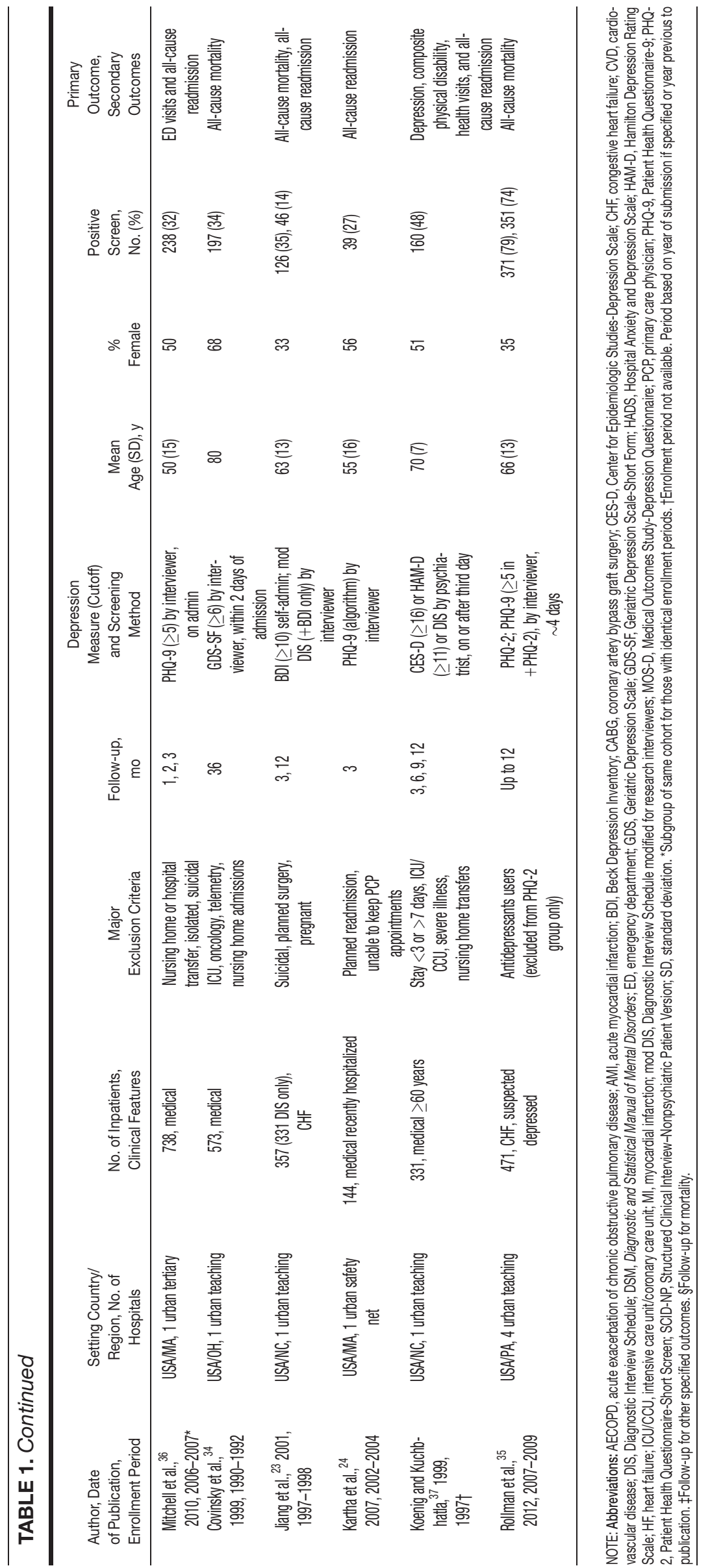




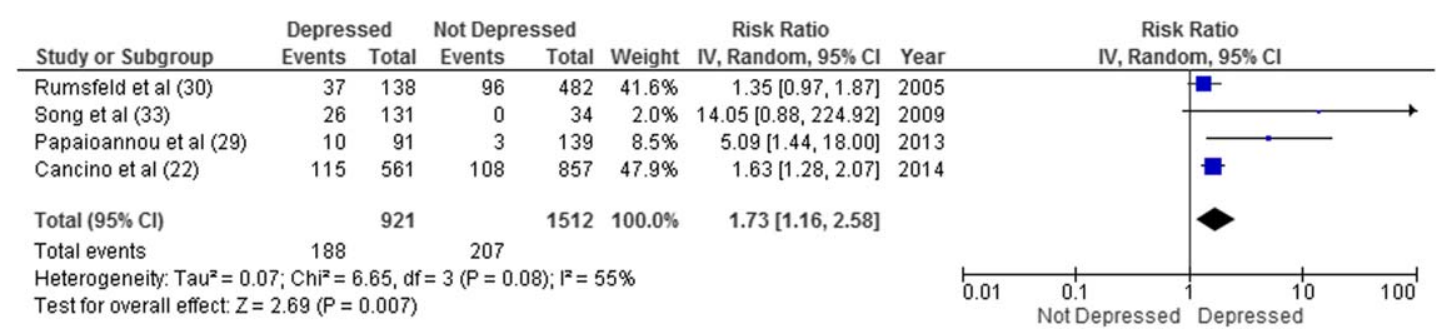

FIG. 1. Risk ratios for 30-day readmission for depressed compared to not depressed patients. Forest plot presents results of the meta-analysis in which the size of each data marker indicates the weight assigned to individuals studies. Abbreviations: Cl, confidence interval; IV, independent variable.

by research assistants or nurses (68\%) or selfadministered (21\%). Most studies examined specific medical patient subgroups (10 cardiac, 3 pulmonary, and 2 elderly). Major exclusion criteria reported were terminal illness (4 studies), unstable condition (6 studies), severe cognitive impairment (5 studies), and suicidal ideation or known depression (4 studies); 1 study enrolled patients with suspected depression (Table 1). Patient cohorts were on average older (range, 50-82 years) (Table 1). Attrition rates for readmission and mortality data were low (average $<1 \%$ among entire sample of studies). All studies scored at least 5 on the Newcastle-Ottawa scale and were thus considered of at least moderate quality (see Supporting Table 1 in the online version of this article).

\section{Prevalence and Recognition of Depressive Symptoms}

The range of depression prevalence in hospitalized medical patients was $14 \%$ to $79 \%$, with a median of $32 \%$ (interquartile range, $27 \%-40 \%$ ) (Table 1 ). In those studies that used a diagnostic interview, the prevalence tended to be lower for major depression, with a median of $17 \%$ (interquartile range, $16 \%-22 \%$ ) (Table 1 ). None of the included studies reported frequency of clinically recognized depression (ie, prior to screening for the study). Only 2 studies assessed the persistence of depression after discharge: 1 reported that depression persisted in $53 \%$ (by screening questionnaire) and 34\% (by diagnostic interview) of patients at 30 days, ${ }^{38}$ whereas the other reported $48 \%$ persistence at 90 days after discharge according to a combined screening method. ${ }^{37}$

\section{Hospital Readmission}

Overall, 8 studies provided readmission data. Among patients discharged from acute care medical wards (4 studies reporting on 5 cohorts), 395 of 2433 $(16.2 \%)$ patients were readmitted within 30 days (Figure 1). Hospitalized patients with depressive symptoms were more likely to be readmitted within 30 days after discharge $(20.4 \%$ vs $13.7 \%$, RR: 1.73 , 95\% CI: $1.16-2.58, P=0.007, I^{2}=55 \%$ ) (Figure $1)$, compared to those without depression. Results were consistent for 90-day readmissions $(39.8 \%$ vs $31.0 \%$, RR: $1.68,95 \%$ CI: $1.13-2.50, \mathrm{P}=0.01, \mathrm{I}^{2}=$ $76 \%, \mathrm{n}=1543$ patients) (see Supporting Figure 2 in the online version of this article) in 6 studies. One individual study examined readmission within 6 months after discharge, but was not poolable in this model, as it presented only hazard ratios and not raw data; however, it did report a 50\% increased risk of readmission in medical inpatients aged $\geq 75$ years (adjusted hazard ratio: $1.50,95 \% \mathrm{CI}: 1.03-2.17, \mathrm{n}=$ 401). ${ }^{39}$

\section{Mortality After Discharge}

Overall, 11 studies provided all-cause mortality data. Among medical patients discharged from acute care in 9 studies, 69 of $3397(2.0 \%)$ patients died within 30 days (Figure 2). Medical patients discharged with depressive symptoms were more likely to die within 30 days $(2.8 \%$ vs $1.5 \%$, RR: 2.13 , 95\% CI: $1.31-$ $3.44, P=0.002, I^{2}=0 \%$ ) (Figure 2) compared to those without depression. Similar results were found for 90 -day mortality $(7.7 \%$ vs $4.1 \%$, RR: $2.01,95 \%$ CI: $1.47-2.76, P<0.001, I^{2}=4 \%, \mathrm{n}=3784$

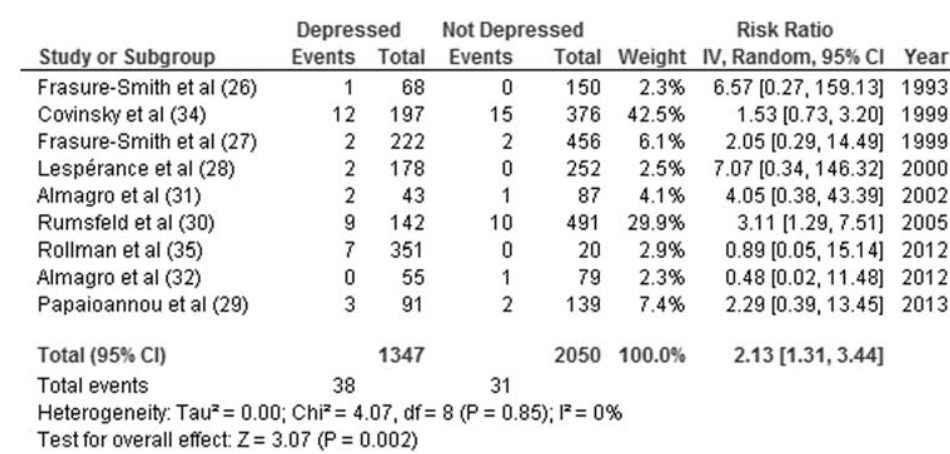

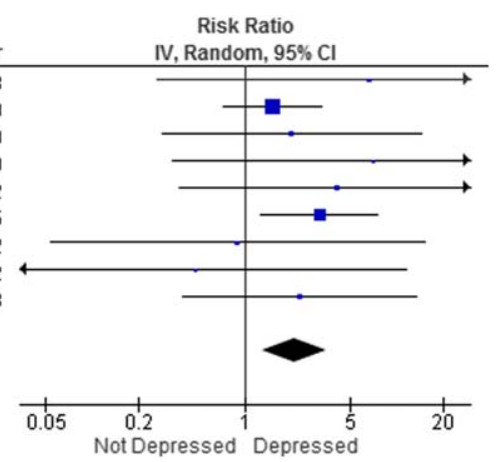

FIG. 2. Risk ratios for 30-day mortality for depressed compared to not depressed patients. Forest plot presents results of the meta-analysis in which the size of each data marker indicates the weight assigned to individuals studies. Abbreviations: Cl, confidence interval; IV, independent variable. 
patients) (see Supporting Figure 3 in the online version of this article) in 11 studies.

\section{ED and PCP Visits}

Four studies examined the use of ED or PCP services within 90 days of discharge, but 3 did not have extractable data for meta-analysis. All showed increased utilization of health services for depressed compared to nondepressed patients after discharge. ${ }^{22,36-38}$ Depressed patients were more likely to visit the ED (adjusted incidence rate ratio: 1.73, 95\% CI: $1.27-2.36),{ }^{36}$ had significantly more medical encounters (eg, PCP, ED visits, hospital admissions, laboratory tests, and home care [mean 2.9 vs $2.6, P=$ $0.05])^{38}$ and had a greater number of ED visits alone (27 vs 15 per 100 patients, $P=0.007)^{22}$ within 30 days of hospital discharge compared to nondepressed patients. Similar results were found at 90 days. ${ }^{36}$

\section{Sensitivity Analyses}

All told, most studies reported a positive association between depression and adverse events, and this was true regardless of how much adjustment for potential confounding had been undertaken by the authors. Although all studies were qualitatively in the same direction, the magnitude of the association varied due to methodological and/or clinical heterogeneity. Sensitivity analysis revealed no overall difference in pooled risk ratios or heterogeneity between Mantel-Haenszel fixed effects versus random effects models or with the addition of 0.5 to cells to permit inclusion of zeroevent data. There was no evidence of publication bias; funnel plots and Egger test results are available upon request. There were no statistically significant differences in the risk associated with depressive symptoms whether studies used Diagnostic and Statistical Manual of Mental Disorders (DSM)-III or DSM-IV criteria, whether the study samples were disease specific or unselected general medical cohorts, whether studies were of moderate or high quality, or regardless of the severity of depressive symptoms.

\section{DISCUSSION}

\section{Summary of Evidence}

We found that depression was common in medical inpatients (about one-third of all patients) and persisted for at least 30 days in up to half of those patients after discharge. We found strong evidence of an association between depressive symptoms and poor short-term prognosis after discharge from the hospital: a $73 \%$ increased risk of readmission and a 2 -fold risk of death within 30 days compared to patients without depressive symptoms with similar results at 90 days.

Our meta-analysis complements a recent systematic review that found concomitant depression to be a risk factor for poor prognosis among inpatients and outpatients with acute coronary syndrome, ${ }^{40}$ and a metaanalysis that demonstrated an increased risk of 2-year mortality among patients with depression after myocardial infarction. ${ }^{41}$ To our knowledge, our study is the first to quantify the short-term postdischarge risks across a diverse group of medical inpatients.

The potential mechanisms underlying the observed relationship between depression and adverse patient outcomes after discharge are likely multiple. We believe there are 2 main possibilities. First, the increased risk associated with depression might be due to residual confounding, even though many of these studies did adjust for extensive lists of comorbidities, 22,24,26,27,29,30,33,35,36,39 including functional status $^{39}$ and prior health services utilization..$^{22,34,36}$ This could occur if other risk factors were not sufficiently adjusted for, such as unrecognized comorbidities or concomitant disability, which are often present among chronically ill patients, ${ }^{42}$ or if depression were a marker of psychosocial risk factors such as anxiety, ${ }^{43}$ stress or poor resiliency, ${ }^{44}$ or low social support, ${ }^{45}$ though a few adjusted for psychosocial factors such as social support ${ }^{26}$ or anxiety. ${ }^{35}$ Confounding could also occur if symptoms of acute illness inflate reports of somatic symptoms of depression on self-report questionnaires. Recent studies on the BDI, found that scores were higher in post-myocardial infarction patients when compared to outpatient controls, ${ }^{46}$ but with no differences between those groups in scores for the BDI-II, ${ }^{47}$ a version with fewer somatic symptom questions.

Second, depression may cause adverse outcomes through indirect or direct pathways. Indirect causation could occur if depression hindered self-care behaviors such as medication adherence. ${ }^{42}$ Depression could also act directly through pathophysiological changes. Some studies have suggested that depression is associated with metabolic abnormalities, including alterations in glucose transport ${ }^{42,48}$ and increased vulnerability to obesity, type 2 diabetes mellitus, and/ or diabetic complications, common conditions among hospitalized patients that also adversely affect postdischarge outcomes. ${ }^{40,48}$

\section{Strengths and Limitations}

This review has multiple strengths. We cast a broad search and included studies that examined a wide range of medical patient subgroups, thus increasing the generalizability of our findings. We identified a general scarcity of studies on this topic and obtained additional unpublished data for 10 of the 18 relevant studies, and our response rate of $34 \%$ is compatible with the $37 \%$ response rate reported for Cochrane reviews when seeking additional data from authors. ${ }^{49}$ Whether examined qualitatively (vote counting of the number of studies that showed an association) or quantitatively (via formal meta-analysis), it seems apparent that there is a clinically important association between depression and postdischarge adverse events, but given the number, quality, and 
heterogeneity of the studies we examined, there may be some ongoing dispute about exactly how strong this association is and the degree of bias contributed by a couple of large studies of the topic.

There are limitations to our review. First, as we did not have individual-level patient data, we could not use metaregression to explore sources of heterogeneity (clinical or methodological) or adjust for confounding, and this likely contributes to observed differences between individual estimates. For instance, the included studies had heterogeneous screening measures and cutoffs; thus, all cases of "depression" in these studies might not be equivalent. Some of the included studies assessed depression early during admission where psychological distress may be greatest; others assessed symptoms closer to discharge. Most studies included patients with specific conditions like heart failure or chronic obstructive pulmonary disease rather than a wide spectrum of medical inpatients. Moreover, few studies adjusted for psychosocial risk factors such as social support, anxiety, and functional status, and only 2 studies assessed the persistence of depressive symptoms after discharge. Second, we did not explore quantitative measures of between-study variation (eg, $\left.I^{2}\right)$, because experts question its utility given the expected heterogeneity in meta-analyses of observational studies. ${ }^{50}$ Third, although the included studies were deemed to be of at least moderate quality, they could be at risk for sources of bias that may not be sufficiently appraised by the current version of the Newscastle-Ottawa scale for observational studies. Finally, we excluded grey literature (eg, conference proceedings or technical reports) that could potentially exclude null findings, although we did contact authors in this field to identify additional unpublished data relevant to this topic.

\section{CONCLUSIONS}

We have confirmed that depressive symptoms are common in hospitalized medical patients, frequently persist after discharge, and may predict greater risk of readmission or death after discharge. Thus, depressive symptoms are an additional marker that clinicians can use to help identify patients in acute care medical settings who may be at increased risk for suboptimal transition back to the community and who may require additional resources after discharge. However, future research is required to evaluate whether treatment of individuals who screen positive for depressive symptoms can reduce 30-day readmission rates, and we are aware of at least 1 relevant ongoing trial (ClinicalTrials.gov, NCT01840826). We believe our study supports calls for clinicians, nurse practitioners, physician assistants, or pharmacists to screen medical inpatients for depressive symptoms prior to discharge and supports the need for trials of interventions (such as multidisciplinary collaborative care that might include inpatient psychiatric teams, advanced practice nurses and social workers) to optimize discharge transitions for these high-risk multimorbid individuals.

\section{Acknowledgements}

The authors thank the following individuals: Dale Storie, MLIS, Saskatchewan Information and Library Services Consortium, Regina, Saskatchewan, Canada, for assistance in the literature search; James A. Hanley, PhD, Department of Epidemiology and Biostatistics, Faculty of Medicine, McGill University, Montreal, Quebec, Canada, for guidance in data recovery methods; Nancy Frasure-Smith, PhD, Department of Psychiatry, McGill University, Department of Psychiatry and Research Centre Hospital Centre, University of Montreal, and Montreal Heart Institute Research Centre, Montreal, Quebec, Canada; Andriana I. Papaioannou, MD, 2nd Respiratory Medicine Department, University of Athens Medical School, Athens, Greece; Konstantinos Kostikas, MD, 2nd Respiratory Medicine Department, University of Athens Medical School, Athens, Greece; and Pere Almagro, MD, Servicio de Medicina Interna, Hospital Universitario Mutua de Terrassa, Terrassa, Barcelona, Spain; as well as Philip G. Jones, MS, Saint Luke's Mid America Heart Institute, Kansas City, Missouri; for their retrieval and contribution of unpublished data.

Disclosures: Ms. Pederson affirms that the manuscript is an honest, accurate, and transparent account of the study being reported with no important omissions. All authors had full access to all of the data (including statistical reports and tables) in the study and can take responsibility for the integrity of the data and the accuracy of the data analysis. Design and conduct of the study: Ms. Pederson, Drs. Majumdar and McAlister. Data acquisition: Ms. Pederson, Ms. Warkentin. Analysis and interpretation of the data and drafting of the manuscript: Ms Pederson, Drs. Majumdar and McAlister. Review of the manuscript: all authors. Study supervision: Drs. Majumdar and McAlister. None of the contributors received compensation for their efforts. Salary support for Ms. Pederson was provided by a CRIO grant from Alberta Innovates-Health Solutions. Drs. McAlister and Majumdar are supported by salary awards from Alberta Innovates-Health Solutions. Dr. McAlister holds the University of Alberta/Capital Health Chair in Cardiology Outcomes Research. Dr. Majumda holds the University of Alberta Endowed Chair in Patient Health Management. The funding sources had no role in the design or conduct of the study; management, analysis, and interpretation of the data; preparation, review, or approval of the manuscript; and decision to submit the manuscript for publication. This work is that of the authors independent of funders. The authors report no conflicts of interest.

\section{References}

1. Jencks SF, Williams MV, Coleman EA. Rehospitalizations among patients in the Medicare fee-for-service program. N Engl J Med. 2009; 360(14):1418-1428.

2. van Walraven C, Bennett C, Ma AJ, Austin PC, Forster AJ. Proportion of hospital readmissions deemed avoidable: a systematic review. CMAJ. 2011;183(7):E391-E402.

3. Fogarty CT, Sharma S, Chetty VK, Culpepper L. Mental health conditions are associated with increased health care utilization among urban family medicine patients. J Am Board Fam Med. 2008;21(5): 398-407.

4. Hansen LO, Young RS, Hinami K, Leung A, Williams MV. Interventions to reduce 30-day rehospitalization: a systematic review. Ann Intern Med. 2011;155(8):520-528.

5. Ayanian JZ. The elusive quest for quality and cost savings in the Medicare program. JAMA. 2009;301(6):668-670.

6. Peikes D, Chen A, Schore J, Brown R. Effects of care coordination on hospitalization, quality of care, and health care expenditures among Medicare beneficiaries-15 randomized trials. JAMA. 2009;301(6): 603-618.

7. Gruneir A, Dhalla IA, van Walraven C, et al. Unplanned readmissions after hospital discharge among patients identified as being at high risk for readmission using a validated predictive algorithm. Open Med. 2011;5(2):e104-111.

8. Kansagara D, Englander H, Salanitro A, et al. Risk prediction models for hospital readmission: a systematic review. JAMA. 2011;306(15): 1688-1698.

9. Dennis M, Kadri A, Coffey J. Depression in older people in the general hospital: a systematic review of screening instruments. Age Ageing. 2012;41(2):148-154.

10. Zhong BL, Chen HH, Zhang JF, et al. Prevalence, correlates and recognition of depression among inpatients of general hospitals in Wuhan, China. Gen Hosp Psychiatry. 2010;32(3):268-275.

11. Cepoiu M, McCusker J, Cole MG, Sewitch M, Belzile E, Ciampi A. Recognition of depression by non-psychiatric physicians-a 
systematic literature review and meta-analysis. J Gen Intern Med. 2008;23(1):25-36.

12. Au AG, McAlister FA, Bakal JA, Ezekowitz J, Kaul P, van Walraven C. Predicting the risk of unplanned readmission or death within 30 days of discharge after a heart failure hospitalization. Am Heart J. 2012;164(3):365-372.

13. Thombs BD, Roseman M, Coyne JC, et al. Does evidence support the American Heart Association's recommendation to screen patients for depression in cardiovascular care? An updated systematic review. PLoS One. 2013;8(1):e52654.

14. Keshavarz H, Fitzpatrick-Lewis D, Streiner DL, et al. Screening for depression: a systematic review and meta-analysis. CMAJ Open. 2013;1(4):E159-E167.

15. Liberati A, Altman DG, Tetzlaff J, et al. The PRISMA statement for reporting systematic reviews and meta-analyses of studies that evaluate health care interventions: explanation and elaboration. Ann Intern Med. 2009;151(4):W65-94.

16. Pignone MP, Gaynes BN, Rushton JL, et al. Screening for depression in adults: a summary of the evidence for the U.S. Preventive Services Task Force. Ann Intern Med. 2002;136(10):765-776.

17. Liu Z, Rich B, Hanley JA. Recovering the raw data behind a nonparametric survival curve. Syst Rev. 2014;3:151.

18. Guyot P, Ades AE, Ouwens MJ, Welton NJ. Enhanced secondary analysis of survival data: reconstructing the data from published Kaplan-Meier survival curves. BMC Med Res Methodol. 2012;12(1): 9.

19. Higgins JPT. Commentary: heterogeneity in meta-analysis should be expected and appropriately quantified. Int J Epidemiol. 2008;37(5): 1158-1160.

20. Wells G, Shea B, Connell D, et al. The Newcastle-Ottawa Scale (NOS) for assessing the quality of nonrandomised studies in metaanalyses. Available at: http://www.ohri.ca/programs/clinical_epidemiology/oxford.htm. Accessed September 1, 2015.

21. Sterne JAC, Becker BJ, Egger M. The funnel plot. In: Rothstein HR, Sutton AJ, Borenstein M, eds. Publication Bias in Meta-analysis: Prevention, Assessment and Adjustments. New York, NY: John Wiley \& Sons; 2006:73-98.

22. Cancino RS, Culpepper L, Sadikova E, Martin J, Jack BW, Mitchell SE. Dose-response relationship between depressive symptoms and hospital readmission. J Hosp Med. 2014;9(6):358-364.

23. Jiang W, Alexander J, Christopher E, et al. Relationship of depression to increased risk of mortality and rehospitalization in patients with congestive heart failure. Arch Intern Med. 2001;161(15):1849-1856.

24. Kartha A, Anthony D, Manasseh CS, et al. Depression is a risk factor for rehospitalization in medical inpatients. Prim Care Companion J Clin Psychiatry. 2007;9(4):256-262.

25. Freedland KE, Carney RM, Rich MW, et al. Depression in elderly patients with congestive heart failure. J Geriatr Psychiatry. 1991; 24(1):59-71.

26. Frasure-Smith N, Lespérance F, Talajic M. Depression following myocardial infarction: impact on 6-month survival. JAMA. 1993;270(15): 1819-1825.

27. Frasure-Smith N, Lespérance F, Juneau M, Talajic M, Bourassa MG. Gender, depression, and one-year prognosis after myocardial infarction. Psychosom Med. 1999;61(1):26-37.

28. Lespérance F, Frasure-Smith N, Juneau M, Théroux P. Depression and 1-year prognosis in unstable angina. Arch Intern Med. 2000; 160(9):1354-1360.

29. Papaioannou AI, Bartziokas K, Tsikrika S, et al. The impact of depressive symptoms on recovery and outcome of hospitalised COPD exacerbations. Eur Respir J. 2013;41(4):815-823.

30. Rumsfeld JS, Jones PG, Whooley MA, et al. Depression predicts mortality and hospitalization in patients with myocardial infarction complicated by heart failure. Am Heart J. 2005;150(5):961-967.
31. Almagro P, Calbo E, Ochoa de Echagüen A, et al. Mortality after hospitalization for COPD. Chest. 2002;121(5):1441-1448.

32. Almagro P, Salvadó M, Garcia-Vidal C, et al. Pseudomonas aeruginosa and mortality after hospital admission for chronic obstructive pulmonary disease. Respiration. 2012;84(1):36-43.

33. Song EK, Lennie TA, Moser DK. Depressive symptoms increase risk of rehospitalisation in heart failure patients with preserved systolic function. J Clin Nurs. 2009;18(13):1871-1877.

34. Covinsky KE, Kahana E, Chin MH. Depressive symptoms and 3 year mortality in older hospitalized medical patients. Ann Intern Med. 1999;130(7):563-569.

35. Rollman BL, Herbeck Belnap B, Mazumdar S, et al. A positive 2-item patient health questionnaire depression screen among hospitalized heart failure patients is associated with elevated 12-month mortality. J Card Fail. 2012;18(3):238-245.

36. Mitchell SE, Paasche-Orlow MK, Forsythe SR, et al. Post-discharge hospital utilization among adult medical inpatients with depressive symptoms. J Hosp Med. 2010;5(7):378-384.

37. Koenig HG, Kuchibhatla M. Use of health services by medically ill depressed elderly patients after hospital discharge. Am J Geriatr Psychiatry. 1999;7(1):48-56

38. Fulop G, Strain JJ, Stettin G. Congestive heart failure and depression in older adults: clinical course and health services use 6 months after hospitalization. Psychosomatics. 2003;44(5):367-373.

39. Büla CJ, Wietlisbach V, Burnand B, Yersin B. Depressive symptoms as a predictor of 6-month outcomes and services utilization in elderly medical inpatients. Arch Intern Med. 2001;161(21):2609_ 2615.

40. Lichtman JH, Froelicher ES, Blumenthal JA, et al. Depression as a risk factor for poor prognosis among patients with acute coronary syndrome: systematic review and recommendations: a scientific statement from the American Heart Association. Circulation. 2014;129(12): 1350-1369.

41. Meijer A, Conradi HJ, Bos EH, Thombs BD, van Melle JP, de Jonge P. Prognostic association of depression following myocardial infarction with mortality and cardiovascular events: a metaanalysis of 25 years of research. Gen Hosp Psychiatry. 2011;33(3): 203-216.

42. Huffman JC, Celano CM, Beach SR, Motiwala SR, Januzzi JL. Depression and cardiac disease: epidemiology, mechanisms, and diagnosis. Cardiovasc Psychiatry Neurol. 2013;2013:695925.

43. Nakamura S, Kato K, Yoshida A, et al. Prognostic value of depression, anxiety, and anger in hospitalized cardiovascular disease patients for predicting adverse cardiac outcomes. Am J Cardiol. 2013;111(10): 1432-1436.

44. Southwick SM, Vythilingam M, Charney DS. The psychobiology of depression and resilience to stress: implications for prevention and treatment. Annu Rev Clin Psychol. 2005;1:255-291.

45. Calvillo-King L, Arnold D, Eubank KJ, et al. Impact of social factors on risk of readmission or mortality in pneumonia and heart failure: systematic review. J Gen Intern Med. 2013;28(2):269-282.

46. Delisle VC, Abbey SE, Beck AT, et al. The influence of somatic symptoms on beck depression inventory scores in hospitalized postmyocardial infarction patients. Can J Psychiatry. 2012;57(12):752-758.

47. Thombs BD, Ziegelstein RC, Pilote L, et al. Somatic symptom overlap in beck depression inventory-II scores following myocardial infarction. Br J Psychiatry. 2010;197(1):61-66.

48. Musselman DL, Betan E, Larsen H, Phillips LS. Relationship of depression to diabetes types 1 and 2: epidemiology, biology, and treatment. Biol Psychiatry. 2003;54(3):317-329.

49. Schroll JB, Bero L, Gøtzsche PC. Searching for unpublished data for Cochrane reviews: cross sectional study. BMJ. 2013;346:f2231.

50. Coory MD. Comment on: heterogeneity in meta-analysis should be expected and appropriately quantified. Int J Epidemiol. 2010;39(3): 932; author reply 933. 